\title{
Strates
}

STRATES Matériaux pour la recherche en sciences sociales

$4 \mid 1989$

Dossier : images réfléchies. Paroles d'un paysan révolutionnaire

\section{Métropole/Métropole. Les géographes et les métropoles d'équilibre}

\section{Marie-Claire Robic}

\section{(2) OpenEdition}

\section{Journals}

Édition électronique

URL : http://journals.openedition.org/strates/4432

DOI : $10.4000 /$ strates.4432

ISSN : $1777-5442$

Éditeur

Laboratoire Ladyss

Édition imprimée

Date de publication : 31 décembre 1989

ISSN : 0768-8067

Référence électronique

Marie-Claire Robic, « Métropole/Métropole. Les géographes et les métropoles d'équilibre », Strates [En ligne], 4 | 1989, mis en ligne le 19 mai 2008, consulté le 08 septembre 2020. URL : http:// journals.openedition.org/strates/4432 ; DOI : https://doi.org/10.4000/strates.4432

Ce document a été généré automatiquement le 8 septembre 2020.

Tous droits réservés 


\title{
Métropole/Métropole. Les géographes et les métropoles d'équilibre $^{1}$
}

\author{
Marie-Claire Robic
}

1 Les géographes ont participé notablement, parmi les spécialistes des sciences sociales, au débat sur la politique des métropoles d'équilibre proposée par la Commission nationale d'Aménagement du territoire dans son premier rapport (CNAT, 1964) et décidée par le Ve Plan. Or la situation d'expertise est alors relativement nouvelle, la catégorie de l'urbain est mal dominée dans la discipline, et les deux termes associés dans le projet paraissent antinomiques. ${ }^{2}$ Comment sont intervenus les géographes : en "romantiques», ainsi que les qualifie quelques années auparavant J. Boudeville (1958) ${ }^{3}$, volontiers sensibles au génie des lieux, à leur unicité, crispés dans un indéfectible attachement au sol et au passé, ou en technocrates partisans d'une action volontariste homogénéisante?

On a pu émettre l'hypothèse que la métropole d'équilibre est l'un des lieux où s'est exprimée une stratégie ambivalente d'intervention territoriale, qui introduit plus de complexité qu'on ne le suppose ordinairement, pour les années soixante notamment, au cœur de la pratique et du discours d'aménagement ${ }^{4}$. Parallèlement, elle serait le lieu d'une inversion des valeurs attribuées à la "métropole», vocable jusqu'alors négatif évoquant la grande ville comme "figure $d u$ désordre». Ce sont ces hypothèses qu'il s'agissait de discuter initialement, à partir de l'analyse des interventions publiques des géographes au cours de l'élaboration de cette politique, - un point de vue circonscrit relevant des représentations et des savoirs plutôt que des pratiques opérationnelles.

C'est sur une perspective historique de plusieurs décennies qu'on a jugé utile de situer les positions des géographes, tant pour ce qui concerne leur analyse de la grande ville que pour leur rapport aux questions d'aménagement spatial. En effet l'étude montre, comme on pouvait s'y attendre, qu'une charnière se situant au tout début des années 1960 a fait basculer les attitudes des géographes. Mais ce n'est pas la transformation d'un corps monolithiquement "ménagiste " ${ }^{5}$ marqué dans son entier 
depuis le "compromis méliniste» par l'idéologie du "paysan-géologue» telle que l'a analysée J-P. Gaudin (1979), - dont nous suivons par ailleurs largement l'interprétation. Il apparaît qu'un courant de géographes prospectifs soucieux, plutôt qu'inquiets, de l'organisation de l'espace national par la grande ville, a coexisté au cours de la première moitié $\mathrm{du} \mathrm{xx}^{\mathrm{e}}$ siècle avec une problématique dominante ruraliste beaucoup plus attachée à une gestion patrimoniale et aux singularités des lieux. Et ce n'est point transformation totale... Mais métropole est polysémique...: la temporalité de cette sensibilité à l'organisation du territoire national par la ville et la mutation des années soixante sont à interroger, sur cette période séculaire, dans les trajectoires croisées d'une Métropole coloniale qui perd son statut et se concentre sur l'Hexagone, et de la polarisation urbaine, métropolisation devenue forme dominante et modèle d'action territoriale.

Visibilité et flou Les interventions des géographes (1960-1965)

Plusieurs analyses récentes ont déjà insisté sur les caractères paradoxaux de l'intervention des géographes dans la politique des métropoles d'équilibre, soit pour déplorer les conséquences d'une implication trop massive dans la recherche appliquée (BURGEL, 1983, par exemple), soit pour souligner à l'inverse que dans cette période 1940-1975 marquée par "l'impatience d'être utile", les savoirs géographiques sont apparus quelque peu obsolètes face à des approches plus conformes aux nouvelles conditions de la production du bâti, et face au développement d'une nouvelle administration, lieu, désormais, de la délimitation du savoir sur l'espace (GAUDIN, 1979). Néanmoins, au cours de ce «rendez-vous manqué » de la géographie active, les géographes ont su réussir une mise à jour partielle en multipliant les études d'armatures urbaines et de polarisation régionale (GAUDIN, 1979). Le considérable développement de la géographie urbaine à partir des années 1950 n'est plus à démontrer ; il est vrai aussi que les géographes ont manifesté une présence visible dans l'élaboration de la politique des métropoles d'équilibre. Mais cette présence est par bien des côtés contestée dans le même temps, si bien que s'impose l'impression qu'un flou accompagne cet engagement manifeste.

La caution de la géographie...

5 Rétrospectivement, la dominante de l'époque paraît être celle d'une caution assez massive accordée par les géographes au parti d'urbanisation concentrée et hiérarchique représenté par la politique des métropoles d'équilibre. Elle opère par une participation polymorphe aux études préalables et à la justification des choix.

6 Une certaine précocité, voire même une anticipation de la commande publique, apparaît d'abord dans les travaux de géographes portant sur l'ensemble des villes françaises multipliés au tournant des années 1960: carte des zones d'influence (publiée par G.CHABOT en 1961, élaborée pendant la décennie précédente), étude du Réseau urbain de la France (COPPOLANI, 1959), investigation systématique du Fait urbain (CARRIERE, PINCHEMEL, 1963), etc. ${ }^{6}$ A ces recherches précoces sur le niveau national s'ajoutent les premiers travaux que le Ministère de la Construction et/ou le Plan commandent aux géographes: étude pionnière de E.JUTLLARD (1961) sur la hiérarchisation des centres, suivie de la série des rapports signés notamment par le géographe M. ROCHEFORT et par J. HAUTREUX, Chef de service au ministère de la Construction, en 1963 et 1964, qui prônent et définissent les «métropoles interrégionales ", futures métropoles d'équilibre. 
7 Par ailleurs, l'argumentation géographique sert de garantie théorique et empirique à la politique en voie d'élaboration, par le biais d'interventions de divers ordres dont, sans viser à l'exhaustivité, on peut indiquer quelques aspects. Rappelons d'abord la paternité de l'analyse de Paris et le désert français, publiée en 1947 et rééditée plusieurs fois (avec nombre de refontes): J.F. GRAVIER, géographe appelé à collaborer au Ministère de la Reconstruction et du Logement auprès de son premier responsable, R. DAUTRY, reste le découvreur de ce déséquilibre fondamental de l'espace français en même temps que le propagandiste d'une mise en valeur très décentralisée.

8 L'invitation à la comparaison systématique entre la France et les pays européens ou les U.S.A., l'appel au changement d'échelle, par la prise en compte des contraintes européennes, participent aussi de cette omniprésence d'un point de vue géographique. S'y ajoutent, légitimation plus théorique, les définitions et analyses prospectives avancées par les géographes sur la région, la ville, le secteur quaternaire. C'est le fait de J.GOTTMANN, armé de sa connaissance du modèle américain. C'est celui de P. GEORGE surtout, autorité incontestée chez les jeunes géographes et respectée par les aménageurs, qui systématise alors la nouvelle version de la région: «La région géographique se définit, en économie avancée, comme la zone de rayonnement et de structuration spatiale d'une ville, la métropole régionale, qui concentre le commerce de collecte et de distribution, domicilie ou transmet les initiatives, administre au sens strict et au sens économique du terme» (1959, p. 181). Il valide aussi le concept de "ville-métropole ", nécessité de l'âge "néo-technique» autant que nouveauté par rapport à la décentralisation industrielle défendue dans le rapport DESSUS de 1949, et justifie la politique des métropoles d'équilibre après examen des questions qu'elle soulève équipement, sélection des villes, relations spatiales (GEORGE, 1968).

$9 \mathrm{Au}$ total, ces interventions réitérées - à côté, certes, de celles des économistes, et s'appuyant elles-mêmes sur la théorie de la polarisation développée par F. PERROUX s'accompagnent d'une frontalité qui se révèle dans les partis pris manifestés lors des études les plus directement liées à l'aménagement. Ainsi, les publications sur l'armature urbaine française opèrent-elles trois réductions sensibles : - réduction de la ville à sa " mission d'encadrement tertiaire du pays", par laquelle elle entre dans un "ensemble hiérarchisé » d'interdépendance (HAUTREUX, LECOURT, ROCHEFORT, 1963, p. 2-3); réduction de la région à son réseau de villes et autonomisation de celui-ci ; « largement autonome quant à son implantation, il doit tirer sa structure rationnelle de ses besoins et de sa logique propre. Pour reprendre les termes de M. le Professeur George, alors que "jadis la région faisait la ville, aujourd'hui, la ville fait la région" ». (HAUTREUX, ROCHEFORT, 1964a, p. 2) ; choix méthodologique d'une identification unique, standardisée, du niveau régional comme du niveau supérieur des villes françaises, la démultiplication des études ne pouvant s'opérer qu'en deçà (id, p. 5).

... sans garantie théorique

10 Cependant ces positions.nouvelles par rapport à la problématique classique de géographie rétrospective et contextuelle, ne rompent pas totalement avec la pratique traditionnelle. Même pour les experts les plus engagés dans la géographie appliquée, l'absence d'une coupure radicale avec cet habitus est source d'un double langage ou d'attitudes en porte-à-faux.

11 Prolongeant un empirisme souvent revendiqué et une certaine image de marque de bon sens, qui a pu favoriser leur écoute dans certains milieux aménagistes, la méfiance à l'égard de la théorie reste prégnante au cœur même de démarches qui sont 
fondamentalement déductives et normatives, tendues vers la rationalité. Ainsi la théorie des lieux centraux, source de la définition de la ville comme centre de relations en interaction avec ses aires de chalandise et avec l'ensemble des autres centres, est-elle à peine citée, sinon rejetée pour cause de géométrisme outrancier. De même est ignorée la théorie de la base économique, tandis que la théorie de la polarisation bénéficie d'une certaine estime, sans préalable critique d'ailleurs. Cette absence d'intérêt, ou plutôt de vigilance, accompagne une utilisation réelle de systèmes théoriques plus ou moins cohérents, peu explicites, peu conscients probablement, apparemment contradictoires avec l'«abdication empiriste " (BOURDIEU, 1984) qui caractériserait les géographes.

La promotion d'un réseau de villes hiérarchisé faisant contrepoids à Paris en province et animant la vie régionale n'est, en outre, pensable que si l'on refoule des analyses géographiques antérieures qui ont conclu à une certaine obsolescence du niveau régional en matière économique. C'est en effet l'une des principales leçons tirées de l'examen génétique des armatures urbaines régionales, notamment celle de l'Alsace :

"Le $\mathrm{XX}^{e}$ siècle démantèle progressivement les bases de son autonomie (...). Pour interpréter toute ville, il ne suffit plus de la replacer dans son réseau régional; il faut saisir les liens de structure qui subordonnent celui-ci à une unité plus vaste constituée par l'Etat ou par la zone d'influence de quelques grands trusts. La véritable unité de structure pour une géographie des villes tend à dépasser le cadre du réseau urbain régional » (ROCHEFORT, 1960, p. 341).

Mais c'est ce qui permet le couplage d'une planification urbaine et d'une planification nationale.

Enfin, cette participation directe à la politique d'aménagement du territoire s'opère sur un mode mineur, sinon à l'époque par le principal protagoniste géographe, M. ROCHEFORT, du moins par P. GEORGE et ses élèves les plus proches qui expriment collectivement, avec la Géographie active (1964), une réserve critique face à l'implication d'intellectuels dans l'application de leur science. Ces réticences, moins éthiques peutêtre, sont partagées par une bonne partie de l'establishment géographique.

Contre le fonctionnalisme urbain frontal

16 La corporation des géographes est en fait partagée car, contrant l'irrésistible ascension des urbanistes, se sont dressés les géographes ruralistes, au nom de la complexité de l'espace rural, ou au nom de l'anarchie des flux qui s'y produirait. En fait, à côté d'attitudes critiques et à côté des comportements strictement défensifs des chercheurs traditionnels, des travaux commandés pour l'aménagement public, tels ceux menés par P. COUTTN et son équipe au Centre d'Etudes Economiques pour le Plan, se sont affrontés plus radicalement aux présupposés des études urbanistiques. L'opposition à ces méthodes d'analyse urbaine se situe dans le modèle défendu par P.COUTIN : couplage de la monographie locale (liée aux enseignements de J.-L. LEBRET) et de la vision d'ensemble, nationale. Pour ce qui est du contenu, les recherches du C.E.E. ont mis l'accent sur les interrelations constitutives du " territoire » rural - selon l'expression de P. COUTIN (1965). Dans le conflit entre l'immobilisme d'un ruralisme traditionnaliste et la technocratie urbaine, ce groupe, un peu marginal dans l'institution géographique, a dû innover en défendant dans les institutions du Plan une voie moyenne d'aménagement de ce qui ne serait plus campagne mais qui, contrôlant l'urbanisation, serait, plutôt que territoire, espace rural. 
17 La critique du "déménagement» du territoire a par ailleurs puisé à deux sources essentiellement :

18 - celle d'un humanisme classique qui regrette " la mort de l'homme-habitant » mais accepterait un renouveau fondé sur l'exaltation de la petite ville par «toute une série d'appuis hiérarchisés"(LE LANNOU, 1967, p. 61);

19 - celle d'un girondisme qui craint la centralisation politique et économique de fait : les métropoles d'équilibre ne risquent-elles pas d'être des "boites à lettres" et non des "centres de décision » (P. PINCHEMEL, J. LABASSE, J. BEAUJEU-GARNIER...). ${ }^{7}$

A l'opposé, l'apologie du renforcement de la concentration parisienne est rare. Pourtant un E. JUILLARD (1967), pionnier en matière d'étude d'armature urbaine nationale, a pu relever les contradictions entre le projet de faire de Paris une «ville mondiale » et la politique des métropoles d'équilibre.

Ruralisme et urbanisme : la grande ville revisitée (1900-1950)

21 Cette participation, tout à la fois visible et floue à la politique d'aménagement urbain du début des années soixante, relaie une tradition secondaire mais réelle quoiqu'encore mal reconnue, d'adhésion à des projets de transformation de l'espace métropolitain par la réorganisation économique régionale et par la grande ville. Au sein d'une géographie urbaine peu développée avant 1950 par rapport à l'étude régionale (BERDOULAY, 1981 ; PINCHEMEL, 1983) et relativement dépréciée par le ruralisme dominant, entendu comme valorisation des valeurs de stabilité sociale attribuées au travail campagnard, il est intéressant d'observer plusieurs traits négligés de l'intérêt des géographes pour la ville, et d'abord pour les métropoles (ROBIC, 1989). ${ }^{8}$

La métropole, ville tentaculaire?

Soulignons d'abord l'attention portée à la grande ville, voire le crédit accordé à la métropole au tournant des $\mathrm{XIX}^{\mathrm{e}}-\mathrm{XX}^{\mathrm{e}}$ siècles et pendant l'entre-deux-guerres. Ainsi les économistes et statisticiens - géographes E. LEVASSEUR et P. MEURIOT - qui soutient une thèse sur la croissance des grandes agglomérations européennes en 1897 - sont partisans du développement des grandes villes tout en reconnaissant les tares morales et les conséquences démographiques qui leur sont imputées : "Le moraliste peut tonner contre les grandes villes; la civilisation ne saurait s'en passer » (LEVASSEUR , 1891, p. 412). La métropole est l'une des têtes de chapitre courantes dans les manuels et essais ${ }^{9}$ : c'est le cas des ouvrages de J. BRUNHES, tant de sa Géographie humaine, première publication du genre (1910) que sa Géographie de la France. C'est aussi une catégorie bien étudiée, puisque R. BLANCHARD, réputé créateur de la géographie urbaine française, a, entre autres, décrit... toutes les futures métropoles d'équilibre !

Du réseau d'étapes aux capitales régionales.

23 Si le même R. BLANCHARD (1911) a établi par son étude sur Grenoble le modèle de la monographie de géographie urbaine, ${ }^{10}$ conservant donc pour l'objet urbain le cadre méthodologique vidalien, il n'en reste pas moins que s'est définie à la même époque une conception de la ville antinomique de l'étude de cas : celle d'une ville en réseau, élément d'un ensemble de villes. L'idée du réseau n'est pas radicalement nouvelle dans la mesure où elle peut s'appuyer sur le système de transport, tel que le décrit par exemple E. RECLUS dans un étonnant article (1895) où, par ailleurs, il aspire à une union de la grande ville avec la campagne environnante. Sur ce plan, l'analyse renvoie à des études nombreuses repérables au cours du deuxième tiers du $\mathrm{XIX}^{\mathrm{e}} \operatorname{siècle~}^{11}$ : les villes, vues à travers leur taille relative et leurs espacements, se comprennent dans un 
réseau d'étapes (network of stations) réglé par la technologie des transports. A l'époque, cette approche de la ville en termes de noeud de communication est encore prégnante dans toutes les études qui valorisent la position, notamment la position fluviale ou maritime.

L'innovation des années 1910, dont P. VIDAL DE LA BLACHE est le principal responsable en France, revient à mettre l'accent indissociablement sur l'organisation de la production et sur l'encadrement territorial dont sont responsables les grandes villes. Il s'agit aux yeux de l'auteur $(1910,1911)$ d'une nouveauté issue notamment de la révolution des transports. La ville, "nodalité", "noeud de rapports", doit se développer en province sous forme de quelques « villes-maitresses (...) entre la ville purement locale et la capitale politique placée trop loin»; mais, puisque l'on assiste parallèlement à la spécialisation régionale, industrielle ou agricole, il s'agit de promouvoir moins des "villes politiques» que des "capitales régionales» chargées d'animer le "faisceau des activités régionales». Ces analyses de la ville comme maillon d'un système régional productif s'insèrent explicitement dans un débat sur la réorganisation de l'espace français : une décentralisation active est prônée à travers un projet de sélection de dix sept villes régionales, appuyé sur une carte des régions correspondantes, dont le ministre du commerce CLEMENTEL s'est inspiré en 1917.

Contradictions des villes-réseaux.

L'émergence de l'économique dans la définition de la grande ville moderne s'accompagne d'une description renouvelée de l'agglomération urbaine, qui hésite entre la critique de l'artifice et l'apologie de l'organisation. La ville moderne, monde en miniature, paraît ainsi à J. BRUNHES et à C. VALLAUX (1921) constituée d'un enchevêtrement de réseaux imbriqués et dominée par le caractère collectif de vies interdépendantes : "Elle doit être réseau de circulation, mieux encore qu'amas de bâtisses " (p. 5-6). Critiquant les métropoles «mangeuses d'hommes» (p. 197), ils prévoient un bouleversement de l'organisation des Etats, en fonction des deux principes nouveaux qu'ils nomment "activité de circulation, division régionale de la production et des échanges". Aussi la réorganisation du maillage territorial doit tendre à un "groupement rationnel " d'ordre économique: "Les divisions territoriales d'un Etat ne seront plus avant tout des divisions de commandement, de l'ordre administratif et politique, car la rapidité des communications rend ce mode de fractionnement de moins en moins nécessaire dans le corps homogène d'une nation organisée: les divisions futures seront faites surtout pour la production et pour l'échange, car ici les communications rapides, loin d'abolir la nécessité des divisions, la rendent de jour en jour plus utiles et plus nécessaires » (p. 407). La dissolution du politique au niveau local et régional au profit de la seule capitale nationale semble aller de pair ici avec une tendance à la valorisation de l'organisation économique d'un espace régional et de l'organisation technique de l'espace intra-urbain. ${ }^{12}$ Malgré les traces d'un provincialisme plus traditionnaliste, c'est ce qui ressort de la géographie politique en œuvre dans l'ouvrage de J. BRUNHES et de P. DEFFONTAINES sur la France (1926).

Les contradictions liées au développement des interactions spatiales dont la grande ville est le centre éclatent dans l'ouvrage de P. LAVEDAN (1936), premier manuel de géographie urbaine, qui, sous un vocable organiciste, rassemble les caractères de la vie urbaine contemporaine. Trois traits généraux, en continuité avec ce qui précède, importent ici. D'abord, l'auteur souligne, spécificité de ce groupement, la nécessaire organisation de la ville: "Par opposition au village, la ville vit continuellement sous une intervention de l'Etat ou de la municipalité. (...) Au sens où l'on parle d'économie dirigée, on 
peut dire que toute la vie urbaine est une vie dirigée » (p. 150). Il s'agit bien, les explications le montrent, d'une organisation technique des réseaux urbains. Plus, la ville ne peut être considérée comme autonome par rapport à l'espace environnant: la région est rapprochée cette fois de l'unité administrative ville, résultat d'une croissance spatiale qui oblige à considérer l'entité " plus grande ville ": «C'est seulement pour le tracé du plan d'extension qu'on est arrivé récemment à la notion d'une région parisienne" rappelle LAVEDAN (p.151). Enfin, la tendance régulatrice relevée est le zoning, voire la "servitude » de zone (p. 160), tels qu'un maréchal Lyautey les a prescrits au Maroc. Au total, et cette conclusion concentre la tonalité pessimiste de toute son analyse, la ville est double contrainte car domination de la nature et, plus nouveau, subordination de l'individu à l'intérêt collectif : «la vie urbaine finit par enclore l'individu comme la nature dans un réseau d'interdictions» (p. 197). L'organisation de cette contrainte serait-elle la solution aux contradictions de l'existence urbaine soulignées par J.BRUNHES et P. DEFFONTAINES lorsqu'ils estiment que " la vie collective dans une ville est un fait contrenature, un perpétuel tour de force, car le peuplement n'est pas proportionnel aux capacités du lieu en air, en eau, en ressources alimentaires, en facilités de communication. (...) Le progrès rend sans cesse la vie des villes plus confortable et plus attirante mais aussi plus précaire» (1926, p. 102)?

Le tournant de 1960 et l'urgence de la métropolisation

Tout se passe comme si le courant urbaniste, minoritaire jusque là, devenait dominant chez les géographes à partir de la fin des années cinquante. Il semble qu'alors émerge un consensus nouveau, banal dans la substitution des valeurs de la croissance à celles de l'équilibre rural, mais profondément intéressant par les processus de renversement du sens accordé à l'espace français et à la grande ville - la métropole.

De la Métropole à l'HexagonePour un monde fini, l'aménagement qualitatif

Le sentiment d'un achèvement de la conquête spatiale du globe, assorti d'une conscience de la rupture historique que cela signifiait, tant du point de vue des modes d'emprise collective sur la terre que de celui des interdépendances mondiales qui en résultent et la fondent, s'est exprimé dès le début de ce siècle chez un géographe comme J. BRUNHES (1909), rare conservationniste de l'époque en France ${ }^{13}$. Se plaçant sur un plan essentiellement intellectuel plutôt que sur celui de la colonisation et du partage du monde opéré à l'époque par les puissances impérialistes européennes, il a développé l'idée que les bords du monde connu (pôles, océans, sommets, intérieur des continents) étant prospectés, il s'agit désormais pour l'homme de conquérir l'univers par la pensée.

C'est toutefois une trentaine d'années plus tard que se sont développés dans les Métropoles des plans visant à mettre en œuvre une intervention territoriale adéquate à ce nouvel état du monde, intériorisée en quelque sorte. La terminologie oppose dès lors au quantitatif, à l'extension, à l'espace vital, l'action qualitative, en profondeur, verticale (au sens littéral), intensive, globale. ${ }^{14} \mathrm{Il}$ en est ainsi du projet du Plan d'Equipement national sous Vichy, qui vise à organiser l'autarcie (BAUDOUI, 1984). A titre programmatique, la géonomie prônée par un M.F. ROUGE à partir de 1944 se fonde aussi sur le constat de finitude du monde: "Entre les deux méthodes possibles, différentes par essence, d'une colonisation intérieure, c'est-à-dire d'une conquête ou d'une organisation, la géonomie est centrée tout entière sur l'idée d'une meilleure utilisation de l'espace, où qu'il soit situé et quelles que soient ses dimensions. Elle vise donc seulement à des changements internes, et uniquement d'ordre qualitatif »(p.52). Si M.F. ROUGE, devenu urbaniste après une 
formation éclectique où la géographie a une bonne part, s'inspire notamment des grands travaux staliniens, c'est à partir du modèle rooseveltien que le géographe J. GOTTMANN a tiré à la même époque sa conviction d'une nécessaire planification territoriale $(1948,1952)$.

Coloniser la France

Les géographes ont pu témoigner d'un mal-développement de la France et prôner une réorganisation qui se fasse au bénéfice de la province et au profit de liaisons interrégionales rompant avec le centralisme. Ainsi P. VIDAL DE LA BLACHE, réputé traditionnaliste et peu enclin à la prospective aménagiste, en parfait universitaire, n'en a pas moins pris parti à plusieurs reprises dans les années 1910-1917 (13) pour des réorganisations majeures de l'espace français, permettant aux potentialités des villes et régions de s'actualiser, permettant surtout, après la réincorporation de l'AlsaceLorraine, à l'empire français de s'adapter à un accroissement sensible de sa force productive. Ce type d'intervention d'ordre géopolitique qui cherche une solution dans l'aménagement du territoire s'est multiplié à partir de la seconde guerre mondiale, à travers les recherches relatives à la décentralisation industrielle entamées sous la Révolution nationale, et à travers les projets de mise en valeur de la France développés au cours des années 1945-1950, lorsque se profile la perspective de modernisation liée au plan Marshall et à la concurrence européenne. La révélation publique des déséquilibres du territoire, suscitée par les titres chocs de J-F. GRAVIER, porte à vrai dire moins sur une sous-exploitation économique du désert français que sur l'état démographique contradictoire de sous-peuplement/congestion. La colonisation intérieure prônée alors consiste dans l'utilisation sur place de la main d'œuvre et de l'ensemble du potentiel productif agricole et industriel, en une politique spatiale active, très décentralisée et à bien des égards ruraliste, hostile à la concentration urbaine.

Le tournant cinquante-soixante rompt brutalement avec ces perspectives de développement territorial. La décolonisation produit une nouvelle urgence, par la vision nouvelle de la finitude: celle de la métropole déchue. La symbolique de l'hexagone semble s'imposer à l'époque en fixant à la fois l'image de la ruche, de la mise au travail, et celle de la fermeture sur soi. Par la symétrie de la figure régulière, qui nait alors, l'hexagone assume aussi la centralisation politique instituée par la cinquième République, et, en matière d'aménagement du territoire, par la création de missions telles que la DATAR (1963). Pour précipiter ce processus de réévaluation de l'image de la France, la guerre d'Algérie a dû être le révélateur de fractures cachées. L'identification du travailleur de province à l'indigène, qui transparaît dans la dénonciation par les régionalistes du colonialisme intérieur à partir de 1962, est violemment rejetée. ${ }^{15}$ Sous le charisme du Général de Gaulle, le repli dans la peau de chagrin se serait-il métamorphosé en concentration sur une nouvelle dimension nationale, dans l'unité symbolisée par l'abstraction géométrique de l'hexagone (ROBIC, 1988 ; COHEN et al. 1989)?

Métropôliser.

Un renversement du sens accordé à la grande ville - à la ville tentaculaire décriée - a accompagné la crispation hexagonale, de telle sorte que sont invalidés les projets de promotion de petites communautés (telles qu'a pu les souhaiter un J-F. GRAVIER), et les perspectives d'une diffusion urbaine généralisée, perspectives qui contribuent à rendre réalistes les propositions de régionalistes de gauche promoteurs d'un développement ascendant (LAFONT, 1967, ROCARD, 1967, etc.). 
Sublimation...

Au cours des années 1960, en effet, la ville devient aux yeux de ses analystes avant tout un lieu de relations, et les études se consacrent essentiellement aux équipements et activités destinés à l'encadrement territorial des populations régionales (PUMAIN, SAINT-JULIEN, 1976). Par là même, les fonctions de production sont éludées ou minorées, ainsi que le relèvent à l'époque des économistes comme C. PONSARD et J. BOUDEVILLE, qui critiquent l'oubli de la base économique lors de la détection des futures métropoles d'équilibre. ${ }^{16}$ Ces choix s'accompagnent naturellement d'une attention particulière à la centralité, à l'accessibilité des villes-métropoles, et à un affinage des centres par le tri des activités qui doivent y être implantées: la métamorphose des centres-villes, accompagnée de leur dédoublement dans nombre de métropoles d'équilibre s'y conforme.

Ces analyses tirent leur sens de la valeur accordée à la révolution qui serait sousjacente. Révolution complète, l'imposition de la fonction urbaine tertiaire est célébrée d'abord comme la fin d'une parenthèse -industrialisation urbaine de l'ère paléotechnique et... banlieues rouges ? - et retour à la vraie nature de la ville (la communication). Elle ouvre aussi l'accès à une ère nouvelle qui serait celle de la société de consommation, marquée par l'accroissement général des niveaux de vie et leur égalisation sur le territoire, sous l'égide de la diffusion urbaine.

Dans cet esprit urbanophile, les fonctions véritablement nobles sont toutefois associées à la seule grande ville : le tertiaire rare ou supérieur, le quaternaire, sont considérés comme l'apanage des métropoles, et précisément, à propos des métropoles d'équilibre à la française, comme moyen de fonder une hiérarchie fonctionnelle objective... et notamment d'attirer les cadres, dont la mobilisation est jugée nécessaire au renversement de l'influence parisienne. ${ }^{17}$

Bref, la nouvelle division spatiale du travail en œuvre, qui favorise la grande ville, est désormais admise et valorisée : témoin l'expression de sublimation fonctionnelle dont use incidemment J.LABASSE pour qualifier le centre d'une métropole européenne accomplie, Dusseldorf. ${ }^{18}$

Polarisation...

37 Garante d'une légitimation scientifique, l'incorporation à la géographie de la théorie économique des pôles de croissance, dont témoigne par exemple le Dictionnaire de la Géographie (1970), a facilité la mutation des valeurs vers l'urbain et l'assentiment des géographes à un processus socio-économique de concentration. Mais cette incorporation s'est faite au prix d'un transfert mal maîtrisé du concept de polarisation économique. Par une déviation engagée dans les travaux de J. BOUDEVILLE (et permise par le relatif flou de la terminologie fondatrice due à F. PERROUX), le concept passe de l'ordre de la production, traduisible par un tableau d'échanges interindustriels, à l'ordre spatial : celui des flux organisés par la ville. Par ailleurs, les mécanismes de domination dont est partie la théorie perrusienne ont pu être transformés en processus d'équilibration et d'homogénéisation, la distinction de PERROUX entre pôle de croissance et pôle de développement étant largement ignorée. ${ }^{19}$

$\mathrm{Au}$ total, dans un contexte de survalorisation des nouveaux processus d'urbanisation, l'homophonie favorise la confusion sémantique entre pôle (polarisation) et métropole (métropolisation). ${ }^{20}$ Cette confusion permet aux géographes d'opérer une mise à jour de leur bagage théorique comme de leur activité de scientifiques. Mais c'est une mise à jour partielle qui consiste à transmuter l'un des concepts classiques de la géographie 
universitaire française par l'opérateur urbain et par la polarisation: la région géographique étant instituée région polarisée et la ville pôle régional, cette mutation a naturalisé dans la discipline l'une des pratiques de l'aménagement du territoire.

Dès lors le relatif accord sur la pertinence de la politique des métropoles d'équilibre, avec ses critiques techniques et déontologiques, de même que l'éludation des problèmes de démocratie et de justice socio-spatiale qui accompagnent sa mise en œuvre ne doivent pas étonner: cette légitimation scientifique du processus de métropolisation se conforte implicitement par le sentiment d'un retour de la ville, après une parenthèse industrielle, à sa vraie nature de lieu de communication, à son statut idéal de Cité ou de Polis.

40 Au terme d'une analyse qui envisage, d'abord, des représentations, on soulignera trois points qui, approfondis, devraient concourir à un éclairage de ce rapport aménagement/ménagement conçu comme ruse pour interroger des pratiques.

41 * Il semble bien que l'approche dominante en matière d'intervention territoriale soit conforme dans ses scansions et dans ses ruptures aux équilibres de la formation sociale à laquelle elle s'applique. En ce sens, le ruralisme des géographes universitaires, prégnant jusqu'au milieu des années 1960, s'accorde bien à une alliance bourgeoisiepaysannat dans la France impériale, dont rend compte l'expression de compromis méliniste. La conversion des années soixante coïncide avec l'intégration de l'agriculture au système capitaliste et une concentration industrielle et financière. Mais des études comparatives seraient à mener dans des formations sociales voisines pour tester la robustesse de ces synchronies d'ensemble.

* A quoi rapporter les courants mineurs détectés à contre-courant des idées majoritaires: d'abord, dans les années 1910-1925, plaidoyer pour la puissance d'organisation issue des grandes villes, qui va de pair avec un projet d'industrialisation, de spécialisation agricole, et de réorganisation politique sous l'égide de groupements intermédiaires, garants d'une solidarité organique; et plus tard, au cours des trente glorieuses, défense de la petite cellule menacée par l'urbanisation...? Surgissent-ils d'une dynamique autonome de recherche? Sont-ils d'abord le fait de marginaux dans l'institution ? ${ }^{21}$ Plus largement, puisent-ils dans des représentations de la société contradictoires avec les représentations dominantes et qui tiendraient par exemple leur origine d'une identification des intérêts du groupe porteur aux intérêts de forces dominées dans l'alliance de classes en cours? Il faudrait entreprendre une sociologie politique très fine des géographes et des experts en général.

* Comment se produit le changement de rapport entre la propension au ménagement et la dévotion à l'aménagement? On peut supposer que des conflits, des traumatismes surviennent. On peut aussi penser que, s'agissant d'une action collective sur le territoire national, une dimension symbolique s'impose, à laquelle la représentation territoriale, l'image mentale de la nation participe fortement. Car ces pratiques admettent nécessairement du sens : la ville, comme la nation, en sont investies. 


\section{BIBLIOGRAPHIE}

Aménagement du territoire et développement régional. Les faits, les idées, les institutions. 1965-1966. Grenoble, I.E.P., 1968, 689 p.

« Aménagement des territoires », 1953. Economie et humanisme. 79.

ANTOINE (S.), WEILL (G.), 1965. « Les métropoles d'équilibre et leur région », Urbanisme. 89, p. 11-19.

BARDET (G.), 1941. Problèmes d'urbanisme, Paris, Dunod, 371 p.

BAUDOUI (R.), 1984. Planification territoriale et reconstruction 1940-1946, Thèse de troisième cycle, Univ. Paris-XII.

BERDOULAY (V.), 1981. La formation de l'école française de géographie (1870-1914), Paris, Bibliomèque Nationale, $245 \mathrm{p}$.

BLANCHARD (R.), 1911, Grenoble, étude de géographie urbaine, Paris, Armand Colin, 162 p.

BOUDEVILLE (J.R.), 1958, « L'économie régionale, espace opérationnel », Cahiers de l'I.S.E.A., série L, 3, $87 \mathrm{p}$.

BOUDEVILLE (J.R.), 1964. « Note sur l'intégration des espaces économiques », Cahiers de l'I.S.E.A., série $\mathrm{L}, \mathrm{n}^{\circ} 14$, p. 5-74.

BOUDEVILLE (J.R.), 1972, Aménagement du territoire et polarisation, Paris, m. th. Génin, 279 p.

BOURDIEU (P.), 1984. Homo academicus, Paris, Ed. de Minuit, 302 p.

BRUNHES (J.), 1909. « Les limites de notre cage », Le Corrrespondant. 10 déc., p. 833-862.

BRUNHES (J.), 1910. La géographie humaine : essai de classification positive ; principes et exemples, Paris, Félix Alcan, 843 p.

BRUNHES (J.), VALLAUX (C.) 1921, La géographie de l'histoire. Géographie de la paix et de la guerre, Paris, Félix Alcan, 716 p.

BRUNHES (J.), 1926, Géographie humaine de la France, Paris, Pion, t. II Géographie politique et géographie du travail, 652 p. (en collab. avec P. DEFFONTAINES).

BRUNET R. et al., 1988. Montpellier Europole, Montpellier, RECLUS, 315 p.

BURGEL (G.), 1983. « Enquête d'identité : le marxisme ? La géographie urbaine ? Villes en parallèle? ", Villes en parallèle, 7, p. 1-3 (présentation du n " Marxisme et géographie urbaine »).

CARRIERE (CF.), PINCHEMEL (P.), 1963, Le fait urbain en France, Paris, Armand Colin, 374 p.

CHABOT (G.), 1961, « Carte des zones d'influence des grandes villes françaises », Paris, C.N.R.S., Mémoires et Documents, t. VIII, p. 139-143.

«Chronique sociale de France », 1965, Métropole régionale, cahier 3-4.

COHEN (J.), GUERRINI (M.C.), MATHIEU (N.), ROBIC (M.C.), 1989, « L'iconographie dans l'aménagement du territoire : changer la donne et/ou donner le change ", Colloque de géographie politique, Paris.

Colloque EDHEC 66, 1966, Métropoles d'équilibre. Ecole des Hautes Etudes Commerciales du Nord. COPPOLANI (J.), 1959, Le réseau urbain de la France, Paris, Les Editions Ouvrières, 80 p. 
COUTIN (P.), 1965, « Les études préparatoires à l'aménagement du territoire rural », Etudes rurales, 16 , p. 78-91.

C.R.E.S.C.O., 1964, Les zones d'influence des villes. Synthèse des études faites en France, Commissariat général du plan d'équipement et de la productivité.

DESSUS (G.), GEORGE (P.), WEULERSSE (J.), 1949. Matériaux pour une géographie volontaire de l'industrie française, Paris, Armand Colin, $180 \mathrm{p}$.

DEZES (M.G.), 1982. Politique urbaine et recherche urbaine. France : 1945-1980. Eléments de bibliographie analytique. S.I., dact., 53 p. $\left(448 \mathrm{n}^{\circ}\right)$.

GAUDIN (J.P.), 1979. L'aménagement de la société. Politiques, savoirs, représentations sociales. La production de l'espace aux XIX ${ }^{e}$ siècle et $\mathrm{XX}^{e}$ siècle, Paris, Anthropos, $425 \mathrm{p}$.

GAUDIN (J.P.), BARRAQUE (B.), 1980. Un bilan des recherches sur la planification urbaine, S.I., dact., $49 \mathrm{p}$.

GAUDIN (J.P.), 1985, L'avenir en plan. Technique et politique dans la prévision urbaine 1900-1930, Seyssel, Champ Vallon, 215 p.

GEORGE (P.), 1959. Questions de géographie de la population, Paris, P.U.F., 231 p. (INED, cahier n 34). GEORGE (P.), 1968, « Les villes-métropoles », Aménagement du territoire et développement régional, vol. 1, p. 135-155.

GEORGE (P.) (éd.), 1970. Dictionnaire de la géographie, Paris, P.U.F., 488 p.

GEORGE (P.), GUGLIELMO (R.), KAYSER (B.), LACOSTE (Y.), 1964. La géographie active, Paris, P.U.F., $394 \mathrm{p}$.

GOTTMANN (J.), 1948. «Changements de structure dans la géographie humaine des Etats-Unis (1948)« Annales de géographie, p. 131-145 et 219-226.

GOTTMANN (J.), 1952, «L'aménagement de l'espace : planification régionale et géographie », Paris, Armand Colin. Cahiers de la Fondation Nationale des Sciences Politiques, 140 p.

GOTTMANN (J.), 1961, Megalopolis. The urbanized northeastern seabord of the United States, Cambridge, Mass., Londres, MIT Press, 779 p.

GRAVIER (J.F.), Paris et le désert français. Décentralisation. Equipement. Population, Paris, Le Portulan, 418 p. (préface de R. Dautry).

GRAVIER (J.F.), 1949, Mise en valeur de la France, Paris, Le Portulan, 384 p.

GUICHARD (O.), 1965, Aménager la France. Inventaire de l'avenir, Paris, Laffont-Gonthier, 274 p.

HAUTREUX (J.), LECOURT (R.), ROCHEFORT (M.), 1963. Le niveau supérieur de l'armature urbaine française. Commissariat général du plan d'équipement et de la productivité, Commission de l'équipement urbain (groupe 1).

HAUTREUX (J.), ROCHEFORT (M.), 1964a, La fonction urbaine dans l'armature urbaine française. Commissariat général du plan d'équipement et de la productivité, Commission nationale de l'Aménagement du Territoire, groupe 5 , ministère de la Construction.

HAUTREUX (J.), ROCHEFORT (M.), 1964b, « Les métropoles et la fonction régionale dans l'armature urbaine française », Construction et aménagement, 17.

JUILLARD (E.), 1961. «Essai de hiérarchisation des centres urbains français actuels ", ministère de la Construction (p. 115-125, in JUILLARD (E.), 1974 : La « région»; contribution à une géographie générale des espaces régionaux, Paris, Ophrys, 230 p.). 
JUILLARD (E.), 1967, « La notion de ville mondiale et le problème de l'avenir de Paris », p. 293-302, in : Mélanges de géographie offerts à M. Orner Tulippe, Gembloux, et dans JUILLARD (E.), 1974, p. 219-230.

LABASSE (J.), 1966, L'organisation de l'espace. Eléments de géographie volontaire. Paris, Hermann, $605 \mathrm{p}$.

LAFONT (R.), 1967, La révolution régionaliste, Paris, Gallimard, 251 p.

LAJUGIE (J.), 1969, « Le schéma français d'armature urbaine », Revue juridique et économique du Sud-Ouest, 18, p. 79-111.

LAJUGIE (J.), DELFAUD (P.), LACOUR (C), 1985, Espace régional et aménagement du territoire, Paris, Dalloz, 987 p. (2 éd.).

LALANNE (L.), 1863, Essai d'une théorie des réseaux de chemin de fer, fondée sur l'observation des faits et sur les lois primordiales qui président au groupement des populations, Comptes rendus des séances de l'Académie des Sciences, t. 57, p. 206-210.

LAVEDAN (P.), 1936, Géographie des villes, Paris, Gallimard, 206 p.

LEBRET (J.L.) et al., 1952-1958, Guide pratique de l'enquête sociale. T. I, Manuel de l'enquêteur. T. II, L'enquête rurale. T. III, L'enquête urbaine. T. IV, L'enquête en vue de l'aménagement régional, Paris, P.U.F.

LEFEBVRE (H.), La production de l'espace, Paris, Anthropos, 487 p.

LE LANNOU (M.), 1967, Le déménagement du territoire. Rêveries d'un géographe. Paris, Seuil, 246 p. (notamment « La petite ville », tiré de Preuves, juin 1965).

LEMAITRE (A.), 1973, La Métropolitée, ou de l'établissement des villes capitales, de leur utilité passive et active, de l'union de leurs parties et de leur anatomie, de leur commerce, etc., Paris, Ed. d'histoire sociale, 195 p., (réimp. de l'édition d'Amsterdam, 1682).

LELAINVILLE (J.), 1913, « Rouen et la région rouennaise », p. 228-260, in : Les divisions régionales de la France, Paris, Félix Alcan, 260 p.

LEVASSEUR (E.), 1891, La population française, Paris, A. Rousseau (t. II, 533 p.).

" Métropoles d'équilibre », 1965. Urbanisme, 89.

MEURIOT (P.), 1897. Des agglomérations urbaines dans l'Europe contemporaine. Essai sur les causes, les conditions, les conséquences de leur développement, Paris, Belin Frères, 475 p.

MONOD (J.), CASTELBAJAC (P. de), L'aménagement du territoire, Paris, P.U.F., 128 p.

MONTIGNY (G.), 1989, Les études urbaines françaises en géographie, sociologie et statistique sociale (1890-1920). Essai d'analyse interdisciplinaire. Thèse EHESS/Paris-X, 3 t., 956 p.

MORAZE (C), 1948, « Géographie et réalité », Annales (Economie, société, civilisation), 1, p. 25-33.

PERROUX (F.), 1950, « Les espaces économiques », Economie appliquée, 1, p. 224-246.

PERROUX (F.), 1955, « La notion de pôle de croissance », Economie appliquée, 1-2 (voir aussi dans L'économie du XXe siècle, 1961).

PERROUX (F.), 1961, L'économie du XXe siècle, Paris, P.U.F.

PIATIER (A.), 1956, « Les attractions commerciales des villes, une nouvelle méthode de mesure », Revue juridique et économique du Sud-Ouest, 4, p. 575-602. 
PINCHEMEL (P.), 1983, « Geographers and the City. A contribution to the history of urban geography in France », p. 295-318, in : PATTEN (J.), (éd.), The expanding city (Mélanges J. GOTTMANN), Londres, Académie Books.

PINCHEMEL (P.), ROBIC (M.C.), TISSIER (J.L.), 1984, Deux siècles de géographie française, Choix de textes, Paris, Comité des travaux historiques et scientifiques, $380 \mathrm{p}$.

PROST (M.A.), 1965, La hiérarchie des villes en fonction de leurs activités de commerce et de service, Paris, Gauthiers-Villars (thèse de doctorat en sciences économiques, 1963).

PUMAIN (D.), SAINT-JULIEN (T.), 1976, « Fonctions et hiérarchies des villes françaises. Etude du contenu des classifications réalisées en France entre 1960 et 1974 », Annales de Géographie, 470, p. $385-440$.

RAUMOLIN (J.), 1984, « L'homme et la destruction des ressources naturelles : la Raubwirtschaft au tournant du siècle », Annales E.S.C., p. 798-819.

RECLUS (E.), 1895, « The évolution of cities », Contemporary Review, janv-juin, p. 246-264.

RHEIN (C), 1988, « Croissance urbaine et peuplement des banlieues. Emploi et structures d'emploi », p. 25-47 in : FOURCAULT (A.), dir., Un siècle de banlieue parisienne, 1859-1964, Guide de recherche, Paris, L'Harmattan, $319 \mathrm{p}$.

RIQUET (P.), 1967, Bibliographie analytique de l'armature urbaine, ministère de l'Equipement et du Logement, $217 \mathrm{p}$.

ROBIC (M.C.), 1982a, « Organisation de l'espace. Contribution à l'étude de la genèse et des significations de l'expression », Documents $n^{\circ}$ 3, GRECO : Histoire du vocabulaire scientifique, Publications de l'Institut National de la Langue Française, p. 69-101.

ROBIC (M.C.), 1982b, « Cent ans avant Christaller... une théorie des lieux centraux », L'Espace géographique, 2, p. 111-123.

ROBIC (M.C.), 1988, « Le panneau Hexagone - France : à l'heur du monde ?», p. 223-230, in : GEOPOINT 86, Avignon, Groupe Dupont, 248 p.

ROBIC (M.C.), 1989, « Perspectives temporelles sur l'émergence de quelques concepts de la géographie urbaine française », Sistemi Urbani (à paraître).

ROCHEFORT (M.), 1960, L'organisation urbaine de l'Alsace, Strasbourg, Publication de la Faculté des Lettres de l'Université de Strasbourg, 384 p.

ROCARD (M.), 1967, Décoloniser la province, Paris, Pons.

RONCAYOLO (M.), 1983, «Logiques urbaines » et « La production de la ville », p. 17-155, in : AGULHON (M.), dir., La ville de l'âge industriel. Le cycle hausmannien, Paris, Seuil, 671 p. (t. IV de Histoire de la France urbaine).

RONCAYOLO (M.), dir., 1985, La ville aujourd'hui. Croissance urbaine et crise du citadin. Paris, Seuil, 671 p. (t. V de Histoire de la France Urbaine).

ROUGE (M.F.), 1944, «Vers une nouvelle discipline : l'organisation de l'espace », Mélanges d'histoire sociale, Paris, p. 45-54.

SAINT-JULIEN (T.), 1985, « Les grandes villes françaises. Un nouveau modèle : pourquoi ? », p. 324-336, in Mélanges offerts à J. Beaujeu-Garnier, Paris, Centre d'analyse de l'espace, université Paris-I, 398 p.

SCHAEFER (F.K.), 1953, « Exceptionnalism in geography : a methodological examination », Annals of the Association of American Geographers, p. 226 - 249. 
S.E.D.E.S., 1966, Les études de métropoles régionales, Commissariat général du Plan d'équipement et de la productivité, 2 vol., 136 p. +28 p.

SIMMEL (G.), 1984, « Métropoles et mentalité », p. 61-76, in : GRAFMEYER (Y.), JOSEPH (L), eds., L'école de Chicago, Naissance de l'écologie urbaine, Paris, Aubier, 335 p. (paru dans Die Grosstadt, Dresde, 1903.

VACHER (A.), 1904, « Montluçon. Essai de géographie urbaine », Annales de Géographie, p. 121-137.

VIDAL DE LA BLACHE (P.), 1910, « Les régions françaises », Revue de Paris, 14 déc., p. 821-849.

VIDAL DE LA BLACHE (P.), 1911, « Sur la relativité des divisions régionales », Athéna, 11 déc., p. 1-8 ; (voir aussi p. 3-14, Les divisions régionales de la France, Paris, Félix Alcan, 1913, 260 p.).

VIDAL DE LA BLACHE (P.), 1917, La France de l'Est (Lorraine, Alsace), Paris, Armand Colin, 280 p.

\section{NOTES}

1. Ce texte reprend, avec quelques mises à jour bibliographiques, une intervention au séminaire « Aménagement et ménagement des territoires » du ministère de l'Urbanisme, du Logement et des Transports, en janvier 1986. Il lui doit sa problématique initiale sur la coexistence, dans toute politique territoriale, de formes d'interventions dénommées aménagistes (aménagistes) opérant comme sur une table rase et de pratiques ménagistes, contextuelles et locales. Une hypothèse seconde s'interroge sur le rôle dans l'aménagement du territoire français des années soixante du transfert dans l'ex-métropole de procédures originellement imaginées et expérimentées dans les colonies. Voir les Dossiers des Séminaires « Techniques, Territoires et Sociétés ", 4 avril 1988 (ministère de l'Equipement et du Logement).

2. De fait, du point de vue de la DATAR, dans la littérature semi-officielle destinée à légitimer sa politique des métropoles d'équilibre, c'est d'abord la métropole qu'il faut exorciser : "Si l'on veut réussir l'opération des métropoles d'équilibre, il faudra que l'opinion comprenne qu'il est possible de vivre agréablement dans les grandes villes de demain " (ANTOINE, WEILL, 1965, p. 19). La grande ville est alors valorisée car elle serait seule porteuse des « services ou qualités supérieures » jugées nécessaires à la croissance générale des besoins et à la jouissance d'une nouvelle élite, celle des cadres (GUICHARD, 1965). Quant à l'équilibre, il suggère volontairement une amélioration du territoire français, au double titre du couple Paris/désert provincial et de l'équipement des régions. Deux termes donc dont l'association est perçue comme choquante, de telle sorte que pour ses promoteurs il doit être clair que l'expression de métropole d'équilibre subsume un pari, un parti pris d'urbanisme nouveau (ANTOINE, WEILL, 1965, p. 15).

3. C'est aussi le diagnostic de C. MORAZE (1948) à propos de la France d'A. DEMANGEON : on trouve une condamnation du même genre (romantisme) à rencontre des géographes américains classiques, défenseurs d'une géographie régionale idiographique, chez l'auteur germano-américain F.K. SCHAEFFER (1953), devenu le porte-drapeau de la nouvelle géographie quantifiée et modélisatrice, précoce admirateur des travaux de W. CHRISTALLER.

4. Cf. l'introduction au séminaire "Aménagement et ménagement des territoires" (M. MARIE, B. BARRAQUE, V. CLAUDE, 1985-1986). A une analyse en termes de succession : aménagement des années 1960 et ménagement des mouvements 
contestataires post-soixante-huitards et des années 1980, l'hypothèse de travail proposée au séminaire suggère une approche dialectique des rapports aménagementménagement : « Ce n'est plus tellement de la production historique de l'aménagement (ou de son altérité réactive) qu'il s'agit ici mais de la production d'un couple aménagement/ménagement, d'une sorte de totalité, où chaque institution même spécialisée dans la gestion de l'une des branches a à penser son contraire ».

5. Terme de l'introduction au séminaire, la frontalité d'approche étant illustrée par exemple par les traits de l'aménagement du territoire exposés par J. BOUDEVILLE dans l'Encyclopaedia Universalis. L'aménagement des années 1960 pourrait être caractérisé par "sa vision essentiellement unilinéaire d'un temps vectorisé par l'innovation technologique et par le règne de la marchandise, sa conception centralisatrice de l'espace hiérarchisé, rationalisé, (...) sa capacité d'occultation, de renvoi sur les marges ou de cantonnement dans des institutions spécialisées d'un certain nombre de phénomènes que les dispositifs intellectuels ont peine à intégrer : sociétés locales, mentalités collectives, minorités, environnement... qui à aucun moment ne sont considérés comme des composants actifs de l'aménagement et des pouvoirs qui le produisent et qu'il produit " (Introduction au séminaire, p. 2).

6. Voir un bilan comparatif de ces recherches dans l'article de D. PUMATN et T. SAINTJULIEN (1976) qui présente aussi une bonne bibliographie. Voir aussi leur bilan de l'évolution récente du système des villes françaises, notamment la singularité des grandes villes : « L'évolution qualitative de l'activité et donc du travail des grandes unités urbaines est peut-être l'expression la plus spectaculaire, et aussi la moins bien connue, des processus qui ont conduit à ce que nous avons appelé l'émergence du modèle "grande ville" " (SAINT-JULIEN, 1985).

7. Voir notamment leurs interventions dans le Colloque EDHEC 66, dans le numéro spécial de la Chronique sociale de France (1965), et dans la revue Urbanisme $\mathrm{n}^{\circ} 89$.

8. Des réévaluations de la conception de la ville et de l'intérêt politique accordé à l'urbain et à une politique d'urbanisme à la fin du $\mathrm{XIX}^{\mathrm{e}}$ siècle et au début du $\mathrm{XX}^{\mathrm{e}}$ siècle dans les sciences sociales et dans les mouvements réformistes sont en cours : voir notamment les thèses de G. MONTIGNY (1989) et de J.-C. GAUDIN (1985), les articles de C. RHEIN (1988) et de M.-C. ROBIC (1989).

9. Le recueil allemand sur la métropole (1903) qui contient un article de F. RATZEL et le célèbre texte de G. SIMMEL, n'est pas recensé dans la Bibliographie Géographique 1903. Il a droit à une brève notice dans la Géographie humaine de J. BRUNHES. RECLUS souligne l'ambivalence de la grande ville, mais lie l'urbanisation au progrès de la civilisation: "To look at our enormous cities, expanding day by day and almost hour by hour (...) and running oui their suckers, like giant octopuses, into the surrounding country, one feels a sort of shudder corne over one, as if in présence of a symptom ofsome strange social malady (...) Yet it is easy to show that this monster growth of the city, the comptez outcome of a multiplicity of causes, is not altogether a morbid growth. If, on the one hand, it constitutes in some of its incidents, a formidable act for the moralist, it is, on the other hand, in its normal development, a sign of healthy and regular évolution. Where the city increases, humanity is progressing; where they diminish, civilisation itselfis in danger » (RECLUS, 1895) p. 246). Cet article est pratiquement repris dans son ouvrage posthume L'homme et la terre, publié entre 1906 et 1908 : cf. extraits dans E. RECLUS, 1982, L'homme et la terre, Paris, FM/La Découverte, t. II, p. 26-47 (Introduction et choix de textes par B. GIBLIN). Voir aussi la traduction et l'analyse de The évolution of cities par J.C. CHAMBOREDON et A. MEJEAN (1988) : « Villes et campagnes selon Elisée Reclus », Cahiers d'économie et sociologie rurales, p. 67-74. 
10. L'expression de géographie urbaine apparait dans les Annales de Géographie en 1904, comme titre d'un article de A. VACHER. La "géographie rurale » n'apparaît qu'en 1926 dans la revue. J. BRUNHES traite du «Boulevard comme fait de géographie urbaine » en 1900.

11. "If the earth were perfectly uniform in the shape ofits relief and the qualifies ofsoil, the towns would occupy, so to speak, an almost geometrical position (...) In the beginning, the step ofman as the natural measure between place and place, (...) was under ordinary conditions, the regular stage between one town and the next. The domestication of animais and, later, the stride of the horse, and then the turn ofthe axle-tree, became the unit of calculation in reckoning the distance between the urban centres of population » (RECLUS, 1895, p. 250-251). Voir auparavant, les écrits de saint-simoniens et d'ingénieurs comme L. LALANNE, spécialiste des réseaux ferrés ou J. REYNAUD (ROBIC, 1982b). Pour l'ensemble du xIX ${ }^{\mathrm{e}}$ siècle, voir RONCAYOLO, 1983. Pour l'émergence de la sociologie et de l'économie urbaines modernes, voir aussi LEMAITRE (1682, réédition 1973) : La Métropolitée... (Information J-F. LANGUMIER). Sur l'analyse des réseaux urbains en géographie voir P. PINCHEMEL, 1983 et ROBIC, 1989.

12. De son côté, pour prévenir des objections contre la grande ville, P. VIDAL DE LA BLACHE (1910) oppose les avantages d'institutions municipales copiées sur les exemples de Birmingham, Manchester et Hambourg.

13. J. RAUMOLIN (1984) souligne qu'au tournant du siècle, seuls des géographes et des géologues ont été sensibles à la précarité des ressources et aux risques de l'économie destructrice.

14. Cf. la terminologie employée par LENINE dans le Développement du capitalisme en Russie. La division espace ouvert/espace fini fonde la présentation de l'ouvrage de J. LABASSE (1966).

15. R. LAFONT (1967) rappelle que cette expression est apparue dans un manifeste occitaniste à propos des événements de Decazeville (1962).

16. P. GEORGE (1968) le remarque bien : « Il est exact de dire que l'accroissement des populations urbaines est davantage aujourd'hui le fait du développement des activités tertiaires que celui des industries. Mais on ne saurait oublier que la base de toute économie reste une activité productive, qu'il ne saurait y avoir de valeur ajoutée sans valeur de base qui est la valeur produite (...). Le premier souci est donc de promouvoir des activités productives techniquement très avancées, voire prospectives, à circuits de capitaux très importants, distribuant des masses salariales élevées et exerçant de ce fait une incitation à l'égard des activités diversifiées » (p. 142).

17. Cf. P. GEORGE (1968) : «On est amené ainsi à faire (...) les bilans des équipements indispensables pour fixer une population exigeante comme l'est une population qui met en balance son installation à Paris ou l'acceptation d'une résidence provinciale, pour attirer des industriels eux-mêmes soumis à diverses attractions » (p. 143).

18. Il rend compte de la différenciation centre-périphérie en indiquant : « Noter la sublimation fonctionnelle déjà très avancée au profit des sièges sociaux, services et banques. La distance de contrôle immédiat des usines atteint facilement $40 \mathrm{~km}$. (cas de Thyssen pour l'usine de Duisbourg)» (LABASSE, 1966, p. 413).

19. Notons encore une sorte de lapsus expressif de l'édulcoration des mécanismes d'asymétrie contenus dans la théorie de la polarisation : « la région vivant par son centre et s'identifiant à lui, la première tâche d'un pays dépourvu de structures spatiales saines sera donc de promouvoir de futures capitales régionales. (...) A partir de là seulement (la connaissance de la structure urbaine présente) il deviendra possible de passer de l'état de fait à 
un schéma amélioré par la mise en place d'équipements nouveaux, favorisant une polarisation cohérente et homogène " (LABASSE, 1966, p. 410 ; souligné par nous). Très critique de l'approche abstraite des économistes, l'auteur est toutefois l'un de ceux qui ont vulgarisé leurs modèles auprès des géographes dans son Organisation de l'espace. 20. Autre histoire d'o... : R. BRUNET (1988) rappelle que le radical pôle (de polein, tourner, d'où la polarisation et le technopôle), se distingue de pôle, lui-même dédoublé en polis (la métropole, la technopole) et en pôlein (vendre, d'où le monopole et éventuellement le technopole); d'où le néologisme d'europole, de genre féminin, construit sur eurein (inventer, trouver) et sur polis.

21. Un groupe catholique pourrait s'individualiser, marginal dans la collectivité radicale de l'entre-deux guerres; de même des non-universitaires comme J. Levainville, etc.

\section{RÉSUMÉS}

Les explications relevant de l'adaptation d'un savoir rétrospectif et contextuel à l'expertise ne rendent pas complètement compte des ambiguïtés de l'intervention des géographes dans l'élaboration de la politique des métropoles d'équilibre. On montre que leur conversion des années soixante à la métropolisation s'ancre dans une tradition mineure de recherches sur l'articulation des relations spatiales modernes par les grandes villes régionales et d'adhésion à l'idée de politiques territoriales ; mais tout s'est passé comme si les enjeux symboliques du repli post-colonial gommaient ceux d'une concentration urbaine " post-industrielle ", à la faveur de la polysémie du terme de métropole, des vertus d'une «polis» retrouvée, et de l'adoption du concept flou de polarisation.

\section{Geographers and the " métropoles d'équilibre » policy}

How geographers happened to get involved in the setting up of the policy called « Métropoles d'équilibre » is rather difficult to trace. In spite of rétrospective explanations taking into account circumstances and knowledge, their part still seems to keep some ambiguous scientific positions. This paper shows that, during the sixties, the new belief in the necessary development of régional capitals came out of a minor research trend concerning the spatial network weaved by urban régional centres and the cmerging idea of territorial policics. But ail happened as if the symbolic challenges of the post-colonial withdrawal were rubbing out those of the postindustrial urban growth. Moreovcr, the use of the polysemous word of « métropole » was jointly recalling the cherished « polis » and agrceing the rather vague concept of polarization.

\section{INDEX}

Mots-clés : métropole d\&\#39, équilibre, histoire de la géographie urbaine, polarisation, division spatiale du travail, décolonisation

Keywords : métropoles, history of urban geography, polarization, spatial division of labour, decolonization 


\section{AUTEUR}

\section{MARIE-CLAIRE ROBIC}

Chargée de recherches au CNRS, elle est rattachée au laboratoire « Epistémologic et histoire de la géographie » (URA 914, CNRS-PARIS 1). Spécialiste de l'histoire de la géographie humaine pendant les $\mathrm{XIX}^{\mathrm{e}}$ et $\mathrm{XX}^{\mathrm{e}}$ siècles, elle a écrit Perspectives temporelles sur l'émergence de quelques concepts de la géographie urbaine française (Sistemi urbani, 2,1989). 